RAD Conference Proceedings, vol. 3, pp. 169-173, 2018

ISSN 2466-4626 (online) | DOI: 10.21175/RadProc.2018.37

www.rad-proceedings.org

\title{
MEGAVOLTAGE COMPUTED TOMOGRAPHY (MVCT) DOSE ASSESSMENT AT DIFFERENT DEPTHS
}

\author{
Sibel Karaca*, Hamit Başaran
}

Erzurum Bölge Eğitim ve Araştırma Hastanesi, Turkey

\begin{abstract}
The aim of the study is to evaluate the point doses measured by different parameters at various depths with MVCT in the Tomotherapy Hi- Art (HT) treatment unit. HT works in two modes: visual modes and therapy modes. The MVCT images are taken in the visual mode. The user can choose the scan length and image pitch value in the visual mode. The system has pitch values called fine, normal and coarse. When the same volume is scanned during the gentry rotation, the scan times of fine, normal, and coarse modes are different from one another. Cheese Phantom is used to evaluate the point doses. The measured values ranged from 0.64 to 2.67 cGy with an average dose of $1.40 c G y$. The lowest MVCT dose is found when 7 slices are scanned with a depth of $20 \mathrm{~cm}$ for 51 seconds. The highest MVCT dose is found when 17 slices are scanned with a depth of $15 \mathrm{~cm}$ for 101 seconds. The measured values are the highest when the fine mode is selected with low depth and high slice. The IGRT method is used before each treatment and can be used more than once if necessary. Therefore, the right mode selection can prevent taking unnecessary doses during MVCT.
\end{abstract}

Key words: Tomotherapy, MVCT, different depths, dose

\section{INTRODUCTION}

In recent years, many radiotherapy (RT) devices also have image-guided radiotherapy (IGRT) [1,2]. Tomotherapy Hi-Art (Accuray Inc., Sunnyvale, CA) (HT) is an image-guided, intensity-modulated radiation therapy (IG-IMRT) system that can obtain a megavoltage computed tomography (MVCT) scan prior to each treatment to minimize daily setup variations $[3,4,5]$. The HT system uses MVCT images for patients initially positioning [6]. MVCT is a sensitive imaging tool that allows anatomical details to be seen. The MVCT system is placed on a ring gantry with a xenon ion-chamber array mounted opposite the radiation source. The accelerator in display $6 \mathrm{MV}$ is tuned to 3.5 MV for the imaging mode [7].This way, the contrast of the soft tissue can be increased during the MVCT scan[8]. Three modes of MVCT image acquisition: coarse, normal and fine, obtained by different pitches (couch movement per gantry rotation 12,8 or $4 \mathrm{~mm}$ ) are available resulting in image reconstruction with inter slice distances of 6, 4 and $2 \mathrm{~mm}$ [9]. Daily MVCT datasets are registered to the treatment planning kilo voltage CT (KVCT) dataset using automated and/or manual image-fusion tools [5]. This allows theverification of patient positioning, target and organ at risk's (OAR's) registration to assess the internal motion (volume changes and the geometric shift), and the reconstruction of the delivered dose [10]. There are some disadvantages as well as the advantages of the daily MVCT view. These disadvantages include the

extra doses given to the patient during the procedure and the prolongation of the treatment period [11].

The aim of the study is to evaluate the point doses measured by different parameters at various depths with MVCT in the Tomotherapy Hi - Art (HT) treatment unit.

\section{MATERIALS AND METHODS}

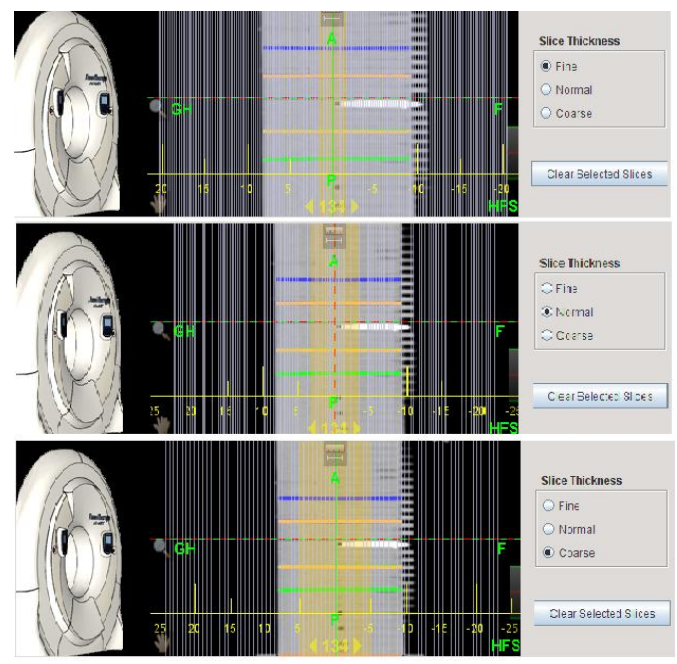

Figure 1. The images of the ion chamber using various pitches (seven slices selected).

*sibeltuzlaci@gmail.com 
S. Karaca and H. Başaran, MVCT dose assessment at different depths, RAD Conf. Proc., vol. 3, 2018, 169-173

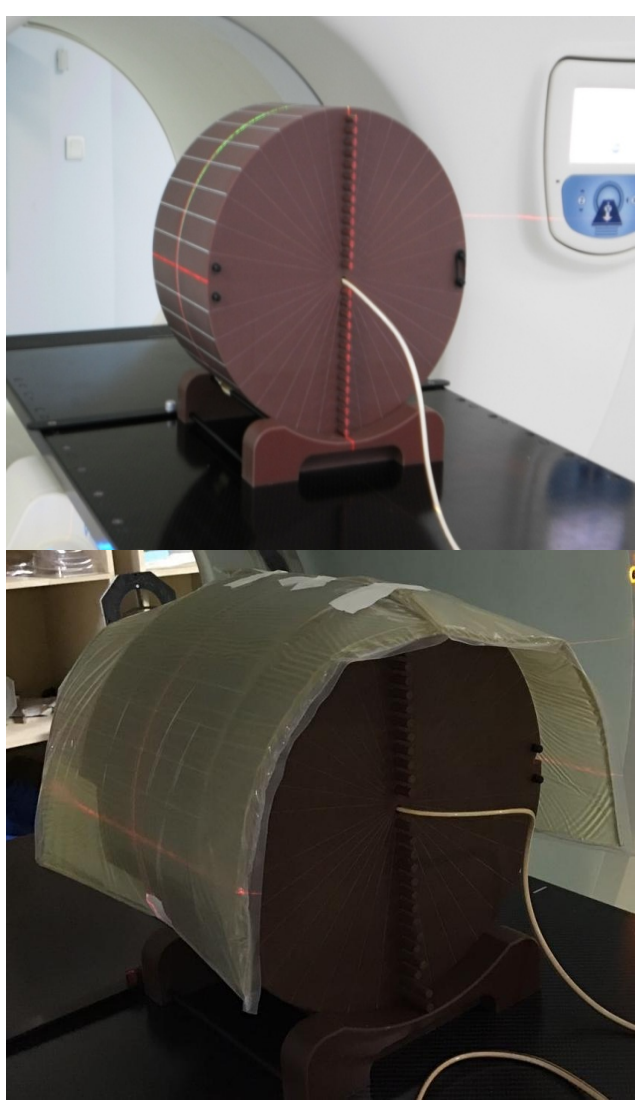

Figure 2. The 'cheese' phantom wrapped with a $0.5 \mathrm{~cm}$ thickness bolus. Exradin A1SL ion chamber (Standard Imaging, Middleton, WI), which is used to verify the absolute dose. The ionization chamber inserted $0.5 \mathrm{~cm}$ below the center of the phantom.

HT is a top-level therapy device with IGRT. The machine works in two modes: visual mode and therapy mode. The nominal amount of energy is $3.5 \mathrm{MV}$ and MVCT images are taken in the visual mode. In the visual mode, MVCT images are visually evaluated and registered with the planning KVCT set either manually or automatically. The system turns all MLCs on and the duration of the gantry period is $10 \mathrm{sec}$ in order to obtain an image during MVCT. The HT system presents two parameters to the user in the visual mode. The first is the scan length selection parameter. The desired volume can be scanned this way. The increase in the scan size also means that the scan time increases. Another parameter is to select the image pitch value. This value represents the length of the nominal slice thickness. The system offers 3 different pitch values. These are called Fine $(2 \mathrm{~mm})$, Normal $(4 \mathrm{~mm})$ and Coarse $(6 \mathrm{~mm})$. When the same slice number is scanned, the fine, normal, and coarse modes' scan length and time are different from one another during the scan (Figure 1).

In this study, point measurements were made at various depths using the Exradin A1SL ion chamber (Standard Imaging, Middleton, WI) connected to TomoElectrometer with Tomotherapy 'cheese' phantom (GammexRMI, Middleton, WI). The cheese is a cylinder of $15 \mathrm{~cm}$ in radius and $15 \mathrm{~cm}$ in length, with a linear series of holes that extends on one face of the phantom for ionization chamber measurements. Exradin A1SL ion chambers $\left(0.057 \mathrm{~cm}^{3}\right)$ were used in measurements. For all measurements, the phantom was positioned onto the HT treatment couch with the ionization chamber inserts disposed along the sagittal direction (perpendicular to the longitudinal axis of the treatment couch) and the ionization chamber inserted $0.5 \mathrm{~cm}$ below the centre of the phantom. The cheese phantom was wrapped with boluses of different thickness $(0.5,1,2,3,4,5 \mathrm{~cm})$ (Figure 2). Boluses of different thickness have been used to obtain different depth measurements. The values read from the electrometer are recorded as Pico Coulomb (PC). The values are calculated according to the required fixed values, the pressure and the temperature of the environment are indicated in Table 1,2 and Figure 2 in terms of CentiGray (cGy).

\section{RESULTS}

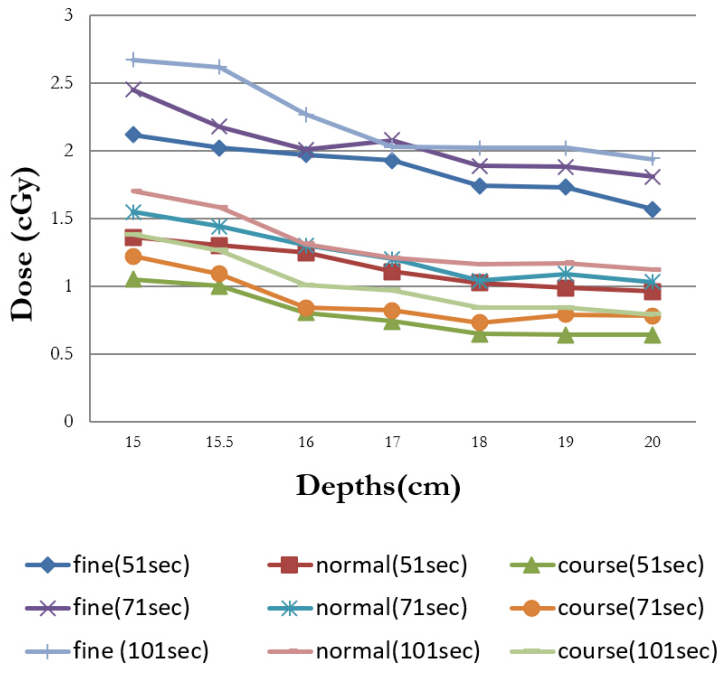

Figure 3. Chart showing measurement dose results at different depths and different mode 
S. Karaca and H. Başaran, MVCT dose assessment at different depths, RAD Conf. Proc., vol. 3, 2018, 169-173

Table 1, 2. MVCT dose values obtained at different depths using Cheese Phantom

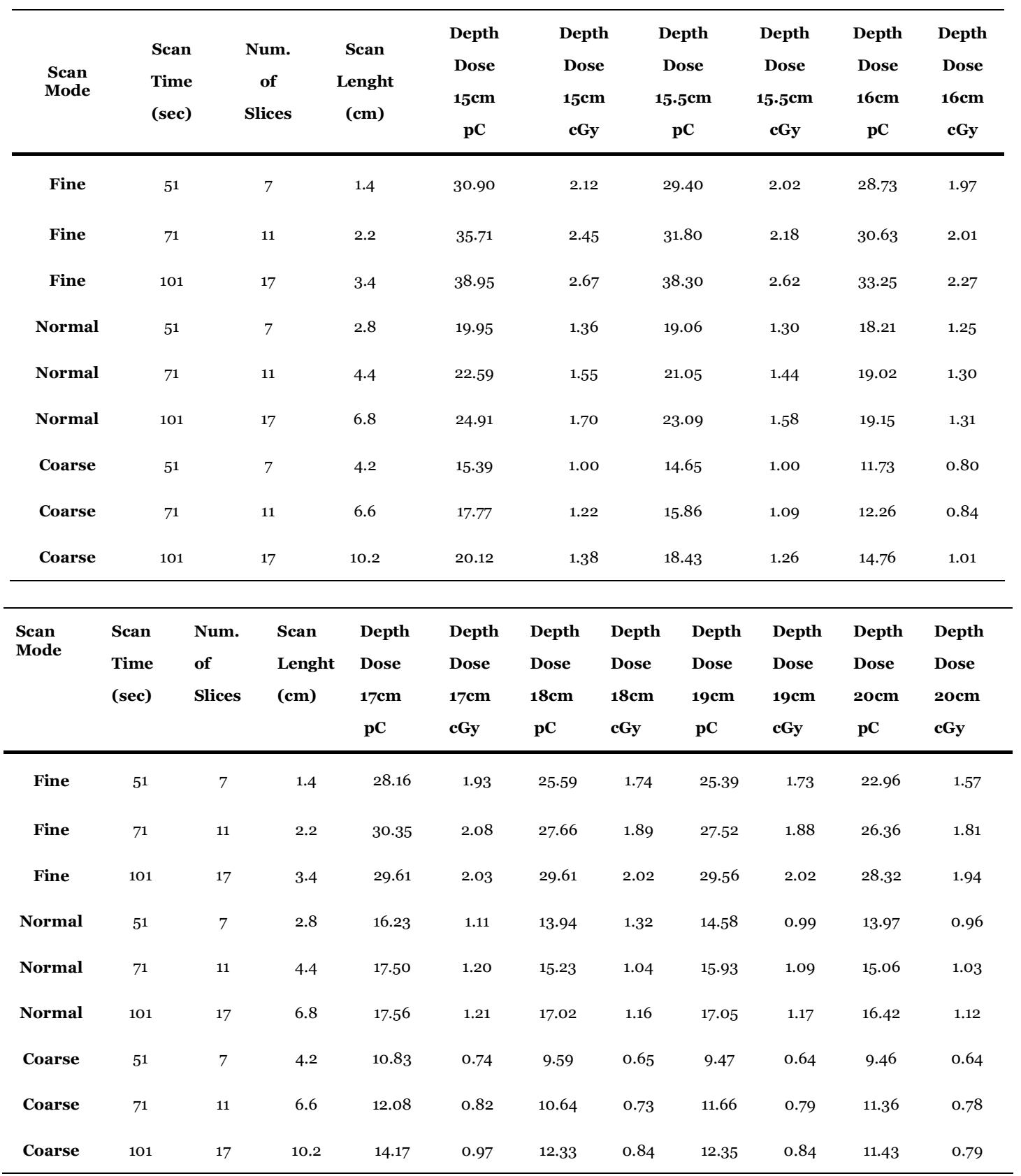

\section{DISCUSSION AND CONCLUSION}

MVCT has become a standard imaging tool for patient localization in HT. The imaging dose from MVCT is often ignored relative to the dose used in patient treatment. However, if a high dose protocol is used on a daily basis. This means that an extra high dose is given to the patient during the treatment process. This research shows that once the doses are analyzed, it can be seen that the measured values are highest when the fine mode with low depth and high slice is selected. Similarly measured values are lowest when the course mode with high depth and low slice is selected.

The image dose is higher in the head and neck than in the pelvis, thorax or abdomen region depending on the tomotherapy procedure used [12,13]. The MVCT doses measured may be different depending on the different screening conditions and different types of phantoms. Xuet al.'s study shows that the MVCT scan dose for the body phantom was between 0.599 and $2.876 \mathrm{cGy}$, while the dose for the head phantom was between 0.913 and 3.231 cGy during each treatment delivery on the tomotherapy device [14]. In similar studies, the absorbed doses of the organs in the head 
S. Karaca and H. Başaran, MVCT dose assessment at different depths, RAD Conf. Proc., vol. 3, 2018, 169-173

and neck and chest region ranged from 1.61-1.89 cGy, while the proportion of organs in the abdomen and pelvis region was between 0.79-1.85 cGy [15, 16]. However, Alaei and Spezi reported that the imaging doses were 4-5 cGy in internal organs and these doses could reach up to $7 \mathrm{cGy}$ on the skin [17].

Tissue characterization solid water phantom (or cheese phantom) with multiple ionization chambers is commonly used in HT dose measurement [9]. In this study, cheese phantom was used for MVCT dose measurements and the measurement results showed that the average dose was $1.40 \mathrm{cGy}$. Similar results were emphasized in other studies. Shah et al. found that the typical imaging dose is approximately $1.5 \mathrm{cGy}$ per imaging HT [18]. Kupelian and Langen's study showed that for the "normal' MVCT scan mode, the MVCT dose ranged from 1 to $1.5 \mathrm{cGy}$ per scan [13].When the perfusion imaging doses of the different IGRT modalities were examined, doses ranged from portal devices 1-10 cGy, kV Cone-beam CT(KV-CBCT) 2-3 cGy, MVCBCT 5-15 cGy, kVFan-Beam CT (kVFBCT) 0.8-2.8 cGy $[19,20,21]$. A number of other reports show that the patient's dose was measured as 0.5-3.0 cGy during the MVCT scan in the HT device $[12,16,22,23]$

The MVCT scans can be acquired in fine, normal and coarse modes. In this study, the lowest MVCT dose is found when scanned in the coarse mode and the highest MVCT dose is found when scanned in the fine mode (coarse mode dose 0.64cGy, fine mode dose $2.67 \mathrm{cGy}$ ). Similarly, Kupelian and Lange found that the fine mode MVCT dose was greater than the normal and coarse mode (coarse mode dose was 0.7cGy, fine mode dose was 3cGy) [13]. Chan at all, in their study was used to cylindrical water phantoms of various diameters for various scanning parameters, including 3 different jaw openings) and couch speeds (fine, normal, and coarse). They have reached $3.1 \mathrm{cGy}$ dose with different jaws in fine mode [24].

In our study, the same protocol was used for different depth measurements. In measurements obtained using the same protocol, higher doses were found at a lower depth. Studies have shown that oversized patients receive significantly higher doses than thin patients during imaging [25]. Protocol selection for thin and oversized patients should be different from each other. This way, better image quality can be obtained.

Another important factor is the timing of patient imaging. In this study, the screening times were 51,71 and $101 \mathrm{sec}$. The scan time may change depending on the scan size and selected treatment mode. In different imaging systems, imaging times are on average 0.1-3 min in portal devices, 2-4 min in KV- CBCT, 2-3 min in MV-CBCT and $15 \mathrm{~min}$ in KV FBCT. The average imaging time is2-3 min in tomotherapy $[19,20]$. The duration of imaging should be calculated taking into consideration the patient size and condition.

The IGRT method should be applied according to clinical targets. The image quality is MVCT if the soft tissue contrast is lower than KCVT [23]. Before selecting the imaging mode, it should be assessed whether the irradiation region is a bone structure or soft tissue. All these evaluations are important for the awareness of pre-imaging.

The IGRT method is used before each treatment and can be used more than once if necessary in the HT device. This study is simulation experiment for visualization of the differences between MVCT doses resulted from changing the size of phantom by helical methods of dose delivery. As a result, it is important to use the best scanning mode for prevent taking unnecessary MVCT doses.

\section{REFERENCES}

1. P. Yadav, R. Tolakanahalli, Y. Rong, B. R. Paliwal, "The effect and stability of MVCT images on adaptive TomoTherapy," J. Appl. Clin. Med. Phys., vol. 11, no. 4, pp. 4-14, Jul. 2010.

DOI: $10.1120 /$ jacmp.v11i4.3229 PMid: 21081878

2. L. Meeks et al., "Performance characterization of megavoltage computed tomography imaging on a helical tomotherapy unit," Med. Phys., vol. 32, no. 8, pp. $2673-2681$, Aug. 2005.

DOI: $10.1118 / 1.1990289$

PMid: 1619379

3. T. R. Mackie et al., "Tomotherapy: a new concept for the delivery of dynamic conformal radiotherapy," Med. Phys., vol. 20, no. 6, pp. 1709 - 1719, Nov-Dec. 1993. DOI: $10.1118 / 1.596958$

PMid: 8309444

4. T. R. Mackie et al., "Image guidance for precise conformal radiotherapy," Int. J. Radiat. Oncol. Biol. Phys., vol. 56, no. 1, pp. 89-105, May 2003.

DOI: $10.1016 /$ So360-3016(03)00090-7

PMid: 12694827

5. S. M. Goddu et al., "Enhanced efficiency in helical tomotherapy quality assurance using a customdesigned water-equivalent phantom," Med. Bio., vol. 54 , no. 19 , pp. $5663-5674$, Oct. 2009.

DOI: $10.1088 / 0031-9155 / 54 / 19 / 001$ PMid: 19724101

6. F. Crop, A. Bernard, N. Reynaert, "Improving dose calculations on tomotherapy MVCT images," J. Appl. Clin. Med. Phys., vol. 13, no. 6, pp. 241 - 253, Sep. 2012.

DOI: $10.1120 /$ jacmp.v13i6.3986 PMid: 23149791

7. L. Minglu, Y. Wang, X. Liao, "Computed Tomography Dose Index Measurement for Hi-ART Megavoltage Helical CT," Radiat. Prot. Dosim., vol. 171, no. 3, pp. $370-374$, Nov. 2016. DOI: $10.1093 / \mathrm{rpd} / \mathrm{ncv} 393$

8. R. Jeraj, T. R. Mackie, J. Balog et al., "Radiation characteristics of helical tomotherapy," Med. Phys., vol. 31, no. 2, pp. 396 - 404, Feb. 2004.

DOI: $10.1118 / 1.1639148$ PMid: 15000626

9. S. Yartsev, T. Kron, D. Van Dyk, "Tomotherapy as a tool in image-guided radiation therapy (IGRT): Theoretical and technological aspects," Biomed. Imaging Interv. J., vol. 3, no. 1, e16, Jan. 2007.

DOI: 10.2349/biij.3.1.e16 PMid: 21614257

10. J. S. Welsh et al., "Clinical implementation of adaptive helical tomotherapy: a unique approach to imageguided intensity modulated radiotherapy," Tech. Cancer Res. Treat., vol. 5, no. 5, pp. $465-479$, Oct. 2006.

DOI: $10.1177 / 153303460600500503$

PMid: 16981789 
S. Karaca and H. Başaran, MVCT dose assessment at different depths, RAD Conf. Proc., vol. 3, 2018, 169-173

11. J. S. Kim et al., "Development of Video Image-Guided Setup (VIGS) System for Tomotherapy: Preliminary Study," Prog. Med. Phys., vol. 24, no. 2, pp. 85 - 91, Jun. 2013.

DOI: 10.14316/pmp.2013.24.2.85

12. J.P. Mege et al., "Evaluation of MVCT imaging dose levels during helical IGRT: comparison between ion chamber, TLD, and EBT3 films," J. Appl. Clin. Med. Phys., vol. 17, no. 1, pp. 143 - 157, Jan. 2016.

DOI: $10.1120 /$ jacmp.v17i1.5774 PMid: 26894346

13. P. Kupelian, K. Langen, "Helical tomotherapy: imageguided and adaptive radiotherapy," Front Radiat. Ther. Oncol., vol. 43, pp. 165 - 180, May 2011.

DOI: $10.1159 / 000322420$ PMid: 21625153

14. S. P. Xu et al., "Measurement and analysis of the imaging dose with megavoltage computed tomography for helical tomotherapy," Chin. J. Cancer, vol. 28, no. 8, pp. 886 - 889, Aug. 2009

DOI: $10.5732 /$ cjc.008.10632 PMid: 19664339

15. K. Son et al., "Evaluation of radiation dose to organs during kilovoltage cone-beam computed tomography using Monte Carlo simulation," J. Appl. Clin. Med. Phys., vol. 15, pp. 295 - 302, Mar. 2014

DOI: $10.1120 /$ jacmp.v15i2.4556 PMid: 24710444

16. M. J. Murphy et al., "The management of imaging dose during image-guided radiotherapy: report of the AAPM Task Group 75," Med. Phys., vol. 34, no. 10, pp. $4041-4063$, Oct. 2007.

DOI: $10.1118 / 1.2775667$

PMid: 17985650

17. P. Alaeiand, E. Spezi, "Imaging dose from cone beam computed tomography in radiation therapy," Phys. Med., vol. 31, no. 7, pp. 647 - 658, Nov. 2015 DOI: 10.1016/j.ejmp.2015.06.003 PMid: 26148865

18. A. P. Shah et al., "Patient dose from megavoltage computed tomography imaging," Int. J. Radiat. Oncol. Biol. Phys., vol. 70, no. 5, pp. 1579 - 1587, Apr. 2008.

DOI: 10.1016/j.ijrobp.2007.11.048
PMid: 18234438

19. A. Bujold et al., "Image-guided radiotherapy: Has it influenced patient outcomes?” Semin. Radiat. Oncol., vol. 22, no. 1, pp. 50 - 61, Jan. 2012.

DOI: 10.1016/j.semradonc.2011.09.001 PMid: 22177878

20. J. Pouliot et al, "Low-dose megavoltage cone-beam CT for radiation therapy," Int. J. Radiat. Oncol. Biol. Phys., vol. 61 , no. 1, pp. $238-241$, Feb. 2005

DOI: 10.1016/j.ijrobp.2004.10.011 PMid: 15736320

21. M. K. Islam et al., "Patient dose from kilovoltage cone beam computed tomography imaging in radiation therapy," Med. Phys., vol. 33, no. 6, pp. 1573 - 1582, Jun. 2006.

DOI: $10.1118 / 1.2198169$

PMid: 16872065

22. J. Balog, G. Olivera, J. Kapatoes, "Clinical helical tomotherapy commissioning dosimetry," Med. Phys., vol. 3o, no. 12, pp. 3097 - 3106, Dec. 2003.

DOI: $10.1118 / 1.1625444$

PMid: 14713076

23. L. J. Forrest et al., "The utility of megavoltage computed tomography images from a helical tomotherapy system for setup verification purposes," Int. J. Radiat. Oncol. Biol. Phys., vol. 6o, no. 5, pp. 1639 - 1644, Dec.2004. DOI: 10.1016/j.ijrobp.2004.08.016 PMid: 15590196

24. M. Chen, E. Chao, W. Lu, "Quantitative characterization of tomotherapy MVCT dosimetry," Med. Dosim., vol. 38 , no. 3 pp. $280-286,2013$.

DOI: 10.1016/j.meddos.2013.02.009 PMid: 23558147

25. S. T. Schindera et al., "Effect of patient size on radiation dose for abdominal MDCT with automatic tube current modulation: phantom study," Am. J. Roentgenol., vol. 190, no. 2, pp. 344, Feb. 2008.

DOI: 10.2214/AJR.07.2891

PMid: 18212190 